From the Department of Haematological Medicine, King's College London and King's College Hospital National Health Services Foundation Trust, London, United Kingdom; Department of Medical Statistics, University of Leiden; Department of Hematology, Leiden University Medical Center, Leiden; Department of Hematology, Radboud University Medical Center Nijmegen, the Netherlands; Division of Clinical Hematology; Hospital de la Sant Creu i Sant Pau, Autonomous University of Barcelona, Spain; Department of Medicine, Hematology and Oncology, University of Freiburg: Department of Bone Marrow Transplantation, University Hospital, Essen; Department of Internal Medicine, University Hospital Leipzig; Department of Bone Marrow Transplantation, University Hospital Hamburg-Eppendorf, Hamburg, Germany; Department of Hematology, Ospedale San Martino, Genova; Policlinico San Matteo Instituti di Ricovero e Cura a Carattere Scientifico, Pavia; Centro Trapianti Midollo, Azienda Ospedaliera S. Giovanni, Torino, Italy; Department of Hematology, Hospital St Louis, Paris; Service de Maladies du Sang, Hospital Claude Huriez, Lille, France; Department of Medicine, Helsinki University Central Hospital, Helsinki, Finland; and Department of Hematology, University Hospital of Leuven, Belgium.

Submitted January 16, 2009; accepted August 13, 2009; published online ahead of print at www.jco.org on December 14, 2009

Written on behalf for the Myelodysplastic Syndromes subcommittee of the

Chronic Leukemia Working Party of the European Group for Blood, and Marrow Transplantation.

Authors' disclosures of potential conflicts of interest and author contributions are found at the end of this article.

Corresponding author: ZiYi Lim, MRCP, FRCPath, Department of Haematological Medicine, Kings College London and Kings College Hospital, Denmark Hill, London, United Kingdom SE5 9RS

e-mail: ziyi.lim@kcl.ac.uk.

(C) 2009 by American Society of Clinical Oncology

0732-183X/10/2803-405/\$20.00

DOI: $10.1200 / J C O .2009 .21 .8073$

\title{
Allogeneic Hematopoietic Stem-Cell Transplantation for Patients 50 Years or Older With Myelodysplastic Syndromes or Secondary Acute Myeloid Leukemia
}

ZiYi Lim, Ronald Brand, Rodrigo Martino, Anja van Biezen, Jürgen Finke, Andrea Bacigalupo, Dietrich Beelen, Agnes Devergie, Emilio Alessandrino, Roel Willemze, Tapani Ruutu, Marc Boogaerts, Michele Falda, Jean-Pierre Jouet, Dietger Niederwieser, Nicolaus Kroger, Ghulam J. Mufti, and Theo M.De Witte

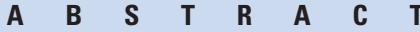

\section{Purpose}

This study was performed to examine the characteristics of transplant activity for patients with myelodysplastic syndromes (MDS) older than 50 years within the European Group for Blood and Marrow Transplantation, and to evaluate the factors predicting outcome within this group of patients.

\section{Patients and Methods}

We performed a retrospective multicenter analysis of 1,333 MDS patients age 50 years or older who received transplantation within the EBMT since 1998. The median recipient age was 56 years, with 884 patients (66\%) age 50 to 60 years and 449 (34\%) patients older than 60 years. There were 811 HLA-matched sibling (61\%) and 522 (39\%) unrelated donor transplants. Five hundred patients (38\%) received standard myeloablative conditioning (SMC), and 833 (62\%) received reduced intensity conditioning $(\mathrm{RIC})$.

\section{Results}

The 4-year estimate for overall survival of the whole cohort was $31 \%$. On multivariate analysis, use of $\mathrm{RIC}$ (hazard ratio [HR], $1.44 ; 95 \% \mathrm{Cl}, 1.13$ to $1.84 ; P<.01$ ) and advanced disease stage at transplantation $(\mathrm{HR}, 1.51 ; 95 \% \mathrm{Cl}, 1.18$ to $1.93 ; P<.01)$ were associated with an increased relapse rate. In contrast, advanced disease stage at transplantation $(\mathrm{HR}, 1.43 ; 95 \% \mathrm{Cl}, 1.13$ to 1.79; $P=.01)$, use of an unrelated donor $(P=.03)$, and $\mathrm{RIC}(\mathrm{HR}, 0.79 ; 95 \% \mathrm{Cl}, 0.65$ to 0.97 ; $P=.03)$ were independent variables associated with nonrelapse mortality. Advanced disease stage at transplantation ( $\mathrm{HR}, 1.55 ; 95 \% \mathrm{Cl}, 1.32$ to $1.83 ; P<.01)$ was the major independent variable associated with an inferior 4-year overall survival.

\section{Conclusion}

Allogeneic hematopoietic stem-cell transplantation remains a potential curative therapeutic option for many older patients with MDS. In this analysis, disease stage at time of transplantation, but not recipient age or the intensity of the conditioning regimens, was the most important factor influencing outcomes.

\section{J Clin Oncol 28:405-411. (C) 2009 by American Society of Clinical Oncology}

\section{INTRODUCTION}

Allogeneic hematopoietic stem-cell transplantation (HSCT) is the only curative treatment for patients with myelodysplastic syndromes (MDS) or secondary acute myeloid leukemia (sAML). Early registry studies demonstrated an adverse association between advanced age and increased nonrelapse mortality (NRM). ${ }^{1-7}$ However, recent improvements in conditioning regimens, supportive care, as well as the introduction of reduced intensity conditioning (RIC) regimens, have allowed the transplantation of older patients. ${ }^{8,9}$ Concurrent improvements in the resolution of HLA typing have facilitated the increased use of unrelated donor transplantation with comparable results to sibling allografts. These developments have led to a significant increase in the number of elderly patients with MDS being referred for allogeneic transplantation. ${ }^{10}$

While much of the drive toward allografting older patients has been made possible by the use of RIC regimens, it remains unclear as to whether it confers an improved overall survival (OS) in elderly patients with MDS. Some studies have suggested that the dose intensity of the conditioning regimen appears to play an important role in relapse-free 
survival post-transplantation for MDS or $\mathrm{AML}^{11,12}$ and recent European Group for Blood and Marrow Transplantation (EBMT) studies in MDS and multiple myeloma have indicated that while the use of RIC is associated with a lower NRM, this is offset by a significantly higher relapse incidence when compared with conventional myeloablative conditioning regimens. ${ }^{6,13}$

With improvements in the quality of life and longevity of the general population, increasing numbers of patients older than 50 years are now being actively treated for MDS. Concurrent with these changes in treatment expectations, many older patients now ask to be considered for an allogeneic HSCT. The challenges facing transplant physicians at present include deciding which older MDS patients should be eligible for allografting, and whether certain older patients may derive greater benefit from receiving an increased dose regimen. Herein, we report on a retrospective multicenter analysis of 1,333 patients with MDS 50 years or older who received transplantation within the EBMT since 1998. The main aims of this study were to examine the characteristics of transplant activity for patients with MDS older than 50 years within EBMT, and additionally to study the factors predicting outcome within this group of patients.

\section{PATIENTS AND METHODS}

Patient details are presented in Table 1. Included in the study were 1,333 patients from 202 centers, with a primary diagnosis of MDS. Only data from patients who underwent a first allogeneic transplantation between January 1998 and October 2006 and who were registered in the EBMT registry were included in this analysis. Data from 295 patients with HLA-matched sibling donors have been reported in a previous EBMT analysis. ${ }^{6}$ All patients were 50 years or older at time of their first allogeneic transplantation. Disease morphology was classified according to the French-American-British (FAB) classification and was documented as separate variables for time of initial diagnosis as well as at time of transplantation. Patients with a diagnosis of chronic myelomonocytic leukemia (CMML) were excluded from the analysis. Three hundred thirteen patients $(24 \%)$ had refractory anemia/refractory anemia with ringed sideroblasts, 471 (35\%) had refractory anemia with excess blasts, 215 (16\%) had refractory anemia with excess blasts in transition, and 334 secondary acute myeloid leukemia $(25 \%)$ at initial diagnosis. Disease status at transplantation was defined as either early ( $<5 \%$ marrow blasts) or advanced $(>5 \%$ marrow blasts).

The median recipient age was 56 years (range, 50 to 74 years) with 884 patients $(66 \%)$ age 50 to 60 years and $449(34 \%)$ age older than 60 years. There were 811 HLA-matched sibling $(67 \%)$ and 522 unrelated donor transplants (33\%). Four hundred nine were matched unrelated donors and 113 were mismatched unrelated donors. Five hundred patients $(38 \%)$ received standard myeloablative conditioning (SMC) and $833(62 \%)$ received RIC as previously defined. ${ }^{6}$ Briefly, RIC included fludarabine plus intermediate doses of one or two alkylating agents or low-dose total body irradiation (2 to $4 \mathrm{~Gy}$ ), with or without anti-thymocite globulin or alemtuzumab. Intermediate doses of alkylating agents were defined as busulphan ( 8 to $10 \mathrm{mg} / \mathrm{kg}$ orally), intravenous melphalan ( 80 to $140 \mathrm{mg} / \mathrm{m}^{2}$ ), intravenous cyclophosphamide (600 to 1,200 $\mathrm{mg} / \mathrm{m}^{2}$ ), or intravenous thiotepa (5 to $10 \mathrm{mg} / \mathrm{kg}$ ).

The median age of the older than 60 cohort was 63 years (range, 60 to 75 ) compared with 54 years (range, 50 to 60 ) for the 50 to 60 cohort. Patients in the older than 60 cohort were more likely to receive a volunteer unrelated donor transplant $(37 \% v 32 \% ; P=.03)$, as well as a RIC regimen $(78 \% v 55 \%$; $P<.01$ ), while patients in the 50 to 60 cohort were more likely to have advanced stage MDS at transplant. Patients receiving RIC were older when compared with SMC recipients (median age, 59 years [range, 50.0 to 74.7 years] $v 54$ years [range, 50.0 to 73.7 years]; $P=.02$ ). However, SMC recipients had more advanced disease stage at both time of diagnosis $(60 \% v 49 \%$;

\begin{tabular}{|c|c|c|}
\hline Characteristic & No. & $\%$ \\
\hline \multicolumn{3}{|l|}{ Median recipient age, years } \\
\hline \multicolumn{3}{|l|}{ Range } \\
\hline $50-60$ & 884 & 66 \\
\hline$>60$ & 449 & 34 \\
\hline \multicolumn{3}{|l|}{ Conditioning } \\
\hline Myeloablative & 500 & 38 \\
\hline Reduced intensity & 833 & 62 \\
\hline \multicolumn{3}{|l|}{ Donor type } \\
\hline HLA-matched sibling & 811 & 61 \\
\hline HLA-matched unrelated & 409 & 31 \\
\hline HLA-mismatched unrelated & 113 & 8 \\
\hline \multicolumn{3}{|l|}{ Stem-cell source } \\
\hline Bone marrow & 256 & 19 \\
\hline Peripheral blood & 1077 & 81 \\
\hline \multicolumn{3}{|l|}{ Transplant period } \\
\hline 1998-2001 & 395 & 30 \\
\hline 2001 onward & 938 & 70 \\
\hline \multicolumn{3}{|c|}{ Interval from diagnosis to transplantation, months } \\
\hline$<6$ & 455 & 34 \\
\hline $6-12$ & 428 & 32 \\
\hline$>6$ & 450 & 34 \\
\hline \multicolumn{3}{|l|}{ Disease stage at diagnosis } \\
\hline RA/RARS & 313 & 24 \\
\hline RAEB & 471 & 35 \\
\hline RAEB-t & 215 & 16 \\
\hline sAML & 334 & 25 \\
\hline \multicolumn{3}{|l|}{ Disease status at transplantation } \\
\hline Early & 557 & 42 \\
\hline Advanced & 688 & 52 \\
\hline Unavailable & 88 & 6 \\
\hline \multicolumn{3}{|l|}{ Cytogenetics } \\
\hline Poor & 89 & 7 \\
\hline Standard & 91 & 7 \\
\hline Good & 225 & 17 \\
\hline Unavailable & 928 & 69 \\
\hline
\end{tabular}

Abbreviations: RA, refractory anemia; RARS, refractory anemia with ringed sideroblasts; RAEB, refractory anemia with excess blasts; RAEB-t, refractory anemia with excess blasts in transition; SAML, secondary acute myeloid leukemia.

$P=.03)$ and at time of transplantation. There was no difference in donor type between RIC and SMC $(P=.15)$.

For graft versus host disease prophylaxis, this variable was not uniformly entered into the EBMT database in most patients, and thus its impact on transplantation outcomes has not been estimated. Fifty-one percent of patients received some form of in vivo T-cell depletion. Due to the retrospective nature of the study, the reason(s) for inclusion in a RIC protocol and exclusion from a SMC in each transplantation group were not known. Informed consent was obtained locally in accordance with the principles laid out in the Declaration of Helsinki and based on specific protocols approved by the respective local and national ethics committees for each transplant center.

\section{Statistical Methods}

End points were assessed on the date of last patient contact, and the final database was updated in October 2006. Analysis focused on NRM, disease relapse, or progression, relapse-free survival (RFS), and OS. Transplantation outcomes were tabulated at 4-years after transplantation, although outcomes at earlier time points are shown in Figure 1 where cumulative incidence estimates are shown in a competing risk context.

The probabilities of RFS and OS estimates from the time of transplantation are identical to the usual Kaplan-Meier estimates and groups were compared using the two-tailed log-rank test. The probability of occurrence of graft 


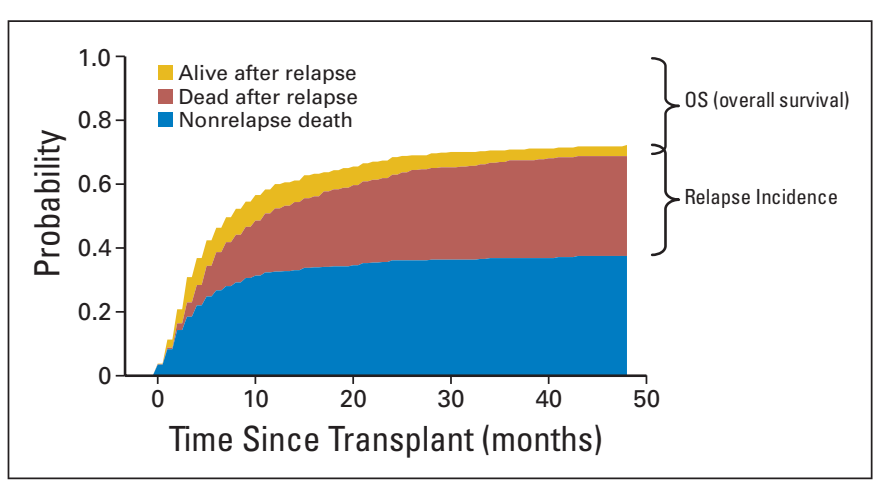

Fig 1. Stacked cumulative incidence curves from a competing risk model evaluating the proportion of patients in a particular state with respect to the presence or absence of relapse, as a function of time after transplant. OS, overall survival.

versus host disease, NRM, and disease relapse or progression was calculated using cumulative incidence estimates, taking into account the appropriate competing risk structure(s). (Cause specific) effect estimates for risk factors were obtained using Cox regression models. The main variables analyzed included donor age, donor status, recipient age group (50 to $60 v>60$ years), FAB disease classification at presentation, disease status at transplantation, type of conditioning regimen, time period of transplantation (1998 to $2001 \mathrm{v}$ 2001-present), time from diagnosis to transplantation ( $<6$ month, 6 to 12 months, and $>12$ months), lines of prior intensive chemotherapy, cytogenetics, donor-recipient cytomegalovirus status, graft type (bone marrow $v$ peripheral blood stem cells). There was sufficient data on cytogenetics in 378 patients (31\%). As such, International Prognostic Scoring System (IPSS) was not included as a variable in this study.

For multivariate analyses, the main covariates were first entered together into the model; with covariates found not to be significant at the .10 level removed from the Cox model in a stepwise backward way. Type of conditioning regimen was held in the model at each step irrespective of its significance since it was the main parameter of interest. Potential interactions between the covariate type of conditioning regimen and the other remaining covariates were tested, adding cross-product terms to the model in a forward stepwise way. Significance tests for all outcomes are based on the usual Cox models which estimate cause-specific hazards and test hazard ratios (HRs) using a likelihood ratio test. However, when we produced survival curves (cumulative incidence estimates) these were based on uni- or bivariate competing risk estimates without underlying model assumptions, but with unbiased curve estimates. When groups were compared according to continuous covariates, the Mann-Whitney $U$ test or Kruskal-Wallis one-way analysis of variance on ranks test were used for differences in medians. According to the group sizes, a $\chi^{2}$ analysis or Fisher's exact test was used to compare categoric covariates. SPSS version 11 (SPSS, Chicago, IL) was used for all statistical analyses.

\section{RESULTS}

\section{OS}

The 4-year estimate OS of the whole cohort was 31\% (Fig 1). At the time of data censure, there were 642 deaths in total. The main identifiable causes of death were relapse in 235 (37\%), secondary malignancy in three $(0.5 \%)$, transplant-related causes 359 (56\%), and other causes 45 (7\%). The 4-year OS estimate of the 50 to 60 years and older than 60 years cohort was $34 \%$ and $27 \%$, respectively, corresponding to a HR of $0.87(P=.23)$. In addition, 4-year estimate OS for patients receiving RIC or SMC was 32\% versus 30\% (HR, 0.97 ; $P=.73$; Fig 2).

\section{Nonrelapse Mortality}

The 4-year estimate NRM was 36\% for all patients. However, patients undergoing RIC had a significantly lower 4-year NRM when compared with those receiving SMC (32\% v 44\%; HR, 0.84; $P=.05)$. While patients older than 60 years had a higher NRM, this was not significantly different from the 50 to 60 age group (4-year estimate: $36 \%$ v 39\%; HR, 1.11; $P=.39)$. When compared with the use of an HLA-matched sibling donor, an HLA-matched or HLA-mismatched

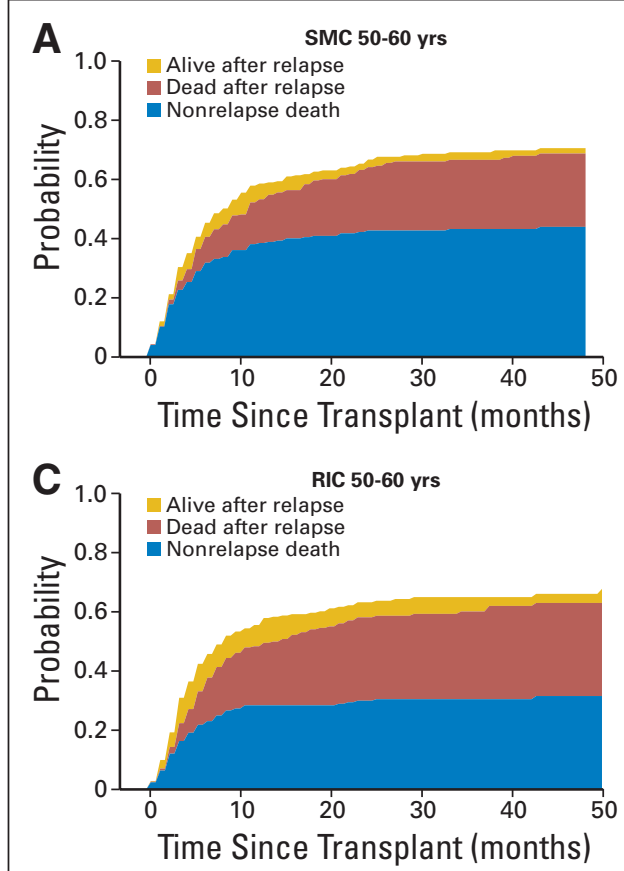

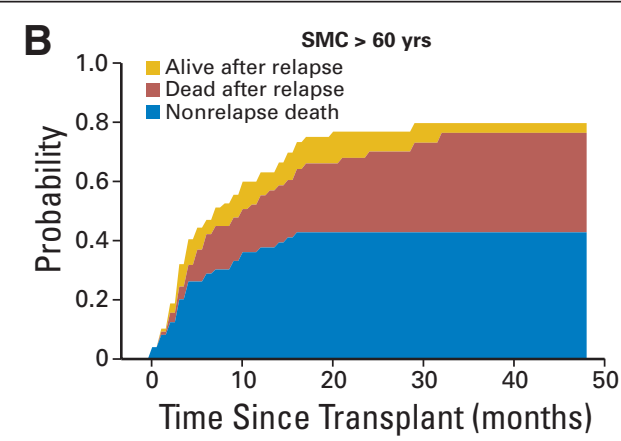

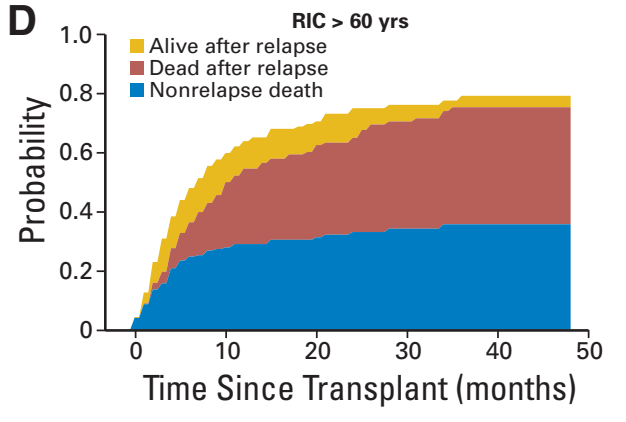

Fig 2. Stacked cumulative incidence curves from a competing risk model with relapse and death as competing risks, with the study population substratified according to $(A)$ age 50 to 60 years, standard myeloablative conditioning (SMC), (B) age $>60$ years, SMC, (C) age 50 to 60 years, reduced intensity conditioning $(\mathrm{RIC})$, and (D) age $>60$ years, RIC. 


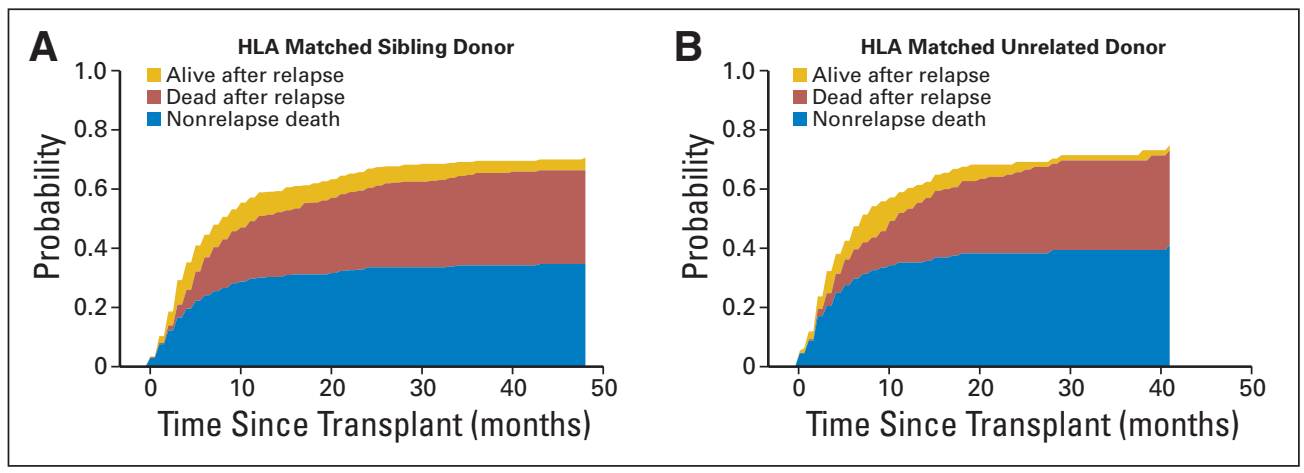

Fig 3. Stacked cumulative incidence curves from a competing risk model with relapse and death as competing risks, with the study population substratified according to donor type: (A) HLA-matched sibling, (B) HLA-matched unrelated donor. unrelated donor was both associated with a higher NRM (4-year estimate: $34 \% v 40 \% v 54 \%$; $P=.02$; Fig 3).

\section{Relapse}

There were 368 relapses in the cohort at time of data censure. Despite more patients with advanced disease receiving SMC, patients with RIC had a higher 4-year relapse rate (41\% v 33\%; HR, 1.39; $P<.01)$. In addition, patients with advanced age had a higher relapse rate ( 50 to 60 years: $32 \% v>60$ years: $41 \%$; HR, $1.32 ; P=.02$ ). There was no significant difference in relapse incidence between patients with either an HLA-matched sibling, HLA-matched or mismatched unrelated donor (4-year estimate: $36 \%$ v 34\% v 38\%; $P=.96$ ).

\section{Cytogenetics}

Based on the IPSS cytogenetic classification, 89 patients were poor risk, 91 standard risk, and 225 favorable risk. There was no significant difference in the choice of conditioning regimens between different cytogenetic risk groups. Patients with available cytogenetic data were more likely to be 50 to 60 years, have an HLA-matched sibling donor, and have less advanced disease stage at time of transplant. On univariate analysis, patients with poor risk cytogenetics had a significantly increased 4-year relapse incidence (71\% v 39\% v 37\%; $P<.01)$ and reduced median OS (8.1 v $19.4 v 30.5$ months; $P<.01)$ when compared with patients with standard or favorable cytogenetics. There was no significant difference in 4-year NRM among groups (42\% v 35\% v 36\%; $P=.24)$.

\section{Multivariate Analysis}

On multivariate analysis of 4-year outcomes (Table 2), use of RIC (HR, $1.44 ; 95 \%$ CI, 1.13 to $1.84 ; P<.01)$ and advanced disease stage at transplantation ( $\mathrm{HR}, 1.51 ; 95 \% \mathrm{CI}, 1.18$ to $1.93 ; P<.01)$ were associated with an increased relapse rate. In contrast, advanced disease stage at transplantation (HR, 1.43; 95\% CI, 1.13 to $1.79 ; P=.01)$, use of RIC (HR, $0.79 ; 95 \% \mathrm{CI}, 0.65$ to $0.97 ; P=.03$ ) and use of an unrelated donor $(P=.03)$ were independent variables associated with NRM. Advanced disease stage at transplantation (HR, 1.55; $95 \% \mathrm{CI}, 1.32$ to $1.83 ; P<.01$ ) was the major independent variable associated with an inferior 4-year OS.

\section{DISCUSSION}

The contribution of recipient age toward the outcomes of allogeneic hematopoietic stem-cell transplantation has been a subject of signifi- cant debate in recent years. The incidence of poor risk MDS is proportionally greater in elderly patients, with increased frequency of sAML, as well as adverse cytogenetic abnormalities. ${ }^{3,7}$ Early registry studies have demonstrated a significant correlation between advanced age and increased NRM. ${ }^{1-4,7,14,15}$ This study is the largest comparing outcomes of patients with MDS older than 50 years undergoing allogeneic HSCT. Of note, while the study is based on the FAB classification of MDS, based on the current WHO classification system, $41 \%$ of the cohort would have AML at time of initial diagnosis. There were inherent imbalances between the cohorts, with patients age 50 to 60 years more likely to receive SMC, with a more advanced disease stage at transplantation, while patients age older than 60 years were more likely to have an unrelated donor. In addition, there are the usual limitations of registry-based studies with the incompleteness of IPSS and cytogenetic data. Nevertheless, on multivariate analysis, there was no significant difference in outcomes between patients aged 50 to 60 years when compared with those older than 60 years. Importantly, at 4 years post-transplantation, $31 \%$ of the cohort was alive, indicating that HSCT is a feasible therapeutic option in a significant proportion of older patients undergoing an allograft for MDS.

With the implementation of reduced intensity conditioning regimens, various groups have demonstrated that older patients with MDS can be successfully allografted. Alyea and colleagues ${ }^{11}$ performed a retrospective analysis of 152 patients with MDS/AML older than 50 years undergoing RIC or myeloablative transplantation. Despite RIC patients being more likely to have unrelated donors and active disease at transplantation, the OS was improved in the RIC group, with the NRM mortality rate being lower for RIC patients but with a higher relapse rate. ${ }^{16}$ More recently, the EBMT reported on the outcomes of 836 patients with MDS who received transplantation with a HLA-identical sibling donor, analyzed according to the type of conditioning received. ${ }^{6}$ Despite the fact that RIC patients were older than patients in the SMC group, the 3-year probability of being progression free was similar, with an increased 3-year relapse rate after RIC offset by a decreased 3-year NRM when compared with SMC. Similarly, in this study, the use of RIC was associated with a higher relapse rate. In contrast, advanced disease stage and the use of an unrelated donor, rather than age or type of conditioning regimen were the only independent factors influencing the NRM.

There is an increasing awareness that transplant physicians need to consider both age as well as the coexistence of other comorbidities in the evaluation of patient suitability for transplantation. This study 


\begin{tabular}{|c|c|c|c|}
\hline Variable & Hazard Ratio & $95 \% \mathrm{Cl}$ & Overall $P^{*}$ \\
\hline \multicolumn{4}{|l|}{ Overall survival } \\
\hline Recipient age, years & & & .42 \\
\hline $50-60$ & Referent & & \\
\hline$>60$ & 1.07 & 0.90 to 1.27 & \\
\hline Conditioning & & & .51 \\
\hline Myeloablative & Referent & & \\
\hline $\mathrm{RIC}$ & 0.95 & 0.80 to 1.11 & \\
\hline Disease stage at transplantation & & & $<.01$ \\
\hline Early & Referent & & \\
\hline Advanced & 1.55 & 1.32 to 1.83 & \\
\hline Donor & & & 0.12 \\
\hline HLA-matched sibling & Referent & & \\
\hline HLA-matched unrelated & 1.25 & 1.02 to 1.47 & .05 \\
\hline HLA-mismatched unrelated & 1.22 & 0.90 to 1.65 & .19 \\
\hline Cytogenetics & & & $<.01$ \\
\hline Standard/favorable risk & Referent & & \\
\hline Poor risk & 1.73 & 1.27 to 2.36 & \\
\hline \multicolumn{4}{|l|}{ Relapse } \\
\hline Recipient age, years & & & .06 \\
\hline $50-60$ & Referent & & \\
\hline$>60$ & 1.42 & 0.99 to 2.04 & \\
\hline Conditioning & & & $<.01$ \\
\hline Myeloablative & Referent & & \\
\hline $\mathrm{RIC}$ & 1.44 & 1.13 to 1.84 & \\
\hline Disease stage at transplantation & & & $<.01$ \\
\hline Early & Referent & & \\
\hline Advanced & 1.51 & 1.18 to 1.93 & \\
\hline Donor & & & .83 \\
\hline HLA sibling & Referent & & \\
\hline HLA-matched unrelated & 1.08 & 0.68 to 1.70 & .75 \\
\hline HLA-mismatched unrelated & 0.99 & 0.62 to 1.59 & .98 \\
\hline Cytogenetics & & & .03 \\
\hline Standard/favorable risk & Referent & & \\
\hline Poor risk & 1.16 & 1.01 to 1.32 & \\
\hline \multicolumn{4}{|l|}{ Nonrelapse mortality } \\
\hline Age, years & & & .82 \\
\hline $50-60$ & Referent & & \\
\hline$>60$ & 0.96 & 0.67 to 1.36 & \\
\hline Conditioning & & & .03 \\
\hline Myeloablative & Referent & & \\
\hline $\mathrm{RIC}$ & 0.79 & 0.65 to 0.97 & \\
\hline Disease stage at transplantation & & & .01 \\
\hline Early & Referent & & \\
\hline Advanced & 1.43 & 1.13 to 1.79 & \\
\hline Donor & & & .03 \\
\hline HLA-matched sibling & Referent & & \\
\hline HLA-matched unrelated & 1.57 & 1.10 to 2.24 & .01 \\
\hline HLA-mismatched unrelated & 1.31 & 0.91 to 1.87 & .14 \\
\hline Cytogenetics & & & .01 \\
\hline Standard/favorable risk & Referent & & \\
\hline Poor risk & 1.18 & 1.04 to 1.34 & \\
\hline $\begin{array}{l}\text { NOTE. Reference variables were ag } \\
\text { stage at transplantation: advanced dis } \\
\text { Abbreviation: RIC, reduced intensit } \\
\text { *Some risk factors in the Cox mode } \\
\text { loss of information; the overall } P \text { val } \\
\text { donor, and cytogenetics denote the } \\
\text { individual } P \text { values denote the } P \text { valu } \\
\text { category. For clarity, the unknown ca } \\
\text { factors have been omitted from the }\end{array}$ & $\begin{array}{l}\text { : } 50-60 \text { years; } \\
\text { ease; donor typ } \\
\text { y conditioning. } \\
\text { contain a cates } \\
\text { ues for age, co } \\
P \text { value of the } \\
\text { of the given } \\
\text { tegories as wel } \\
\text { table. }\end{array}$ & $\begin{array}{l}\text { conditioning: } R \\
\text { e: HLA-matche } \\
\text { gory for unknov } \\
\text { nditioning, dise } \\
\text { complete risk } \\
\text { contrasts to the } \\
\text { las the nonsigr }\end{array}$ & $\begin{array}{l}\text { RIC; disease } \\
\text { ed sibling. } \\
\text { wn to avoid } \\
\text { ease stage, } \\
\text { factor; the } \\
\text { e reference } \\
\text { nnificant risk }\end{array}$ \\
\hline
\end{tabular}

suggests again that advanced age per se, should not be a contraindication for allografting in MDS. Assessment scores such as the Charlson comorbidity score, or more recently, the specifically developed hematopoietic cell transplantation comorbidity index may help to improve patient selection, ${ }^{17-19}$ and comorbidity indices should be incorporated as part of transplant registry data collection forms for future studies.

The single most important prognostic variable in our study was the disease stage at time of transplantation, with patients with more than $5 \%$ blasts at time of transplantation having a significantly inferior RFS and OS. Our findings are consistent with reported data indicating that in MDS/AML, a low pretransplant tumor burden is essential for the success of the allograft. ${ }^{20-23}$ Intensive chemotherapy is effective in inducing complete remissions in $15 \%$ to $65 \%$ of patients. ${ }^{23,24}$ However, these remissions are often short-lived due to subsequent disease relapse. While it may appear logical to pretreat patients with intensive chemotherapy to reduce the disease burden, controversy remains about the effectiveness of this approach, as it is unclear if intensive chemotherapy merely identifies a subgroup of patients with more chemotherapy-responsive treatment.

The observation that patients receiving SMC have a lower relapse incidence when compared with patients receiving RIC in our cohort supports existing evidence suggesting that the dose intensity of the conditioning regimen has an important influence on the posttransplant remission rates. ${ }^{12}$ However, recent data from Scott et al ${ }^{22}$ on 150 patients with MDS/sAML receiving RIC or SMC regimens demonstrated that the overall and progression-free survival rates were similar for patients with chemotherapy-induced remissions irrespective of conditioning intensity, suggesting that graft versus leukemia effects may be more important in preventing progression in patients in chemotherapy-induced remissions at the time of transplantation. In contrast, Aylea and collegues ${ }^{11}$ have shown that increasing the dose intensity of RIC regimens reduces the relapse incidence among patients with either MDS or AML, albeit with increased treatment related toxicity. Indeed, there is evidence to suggest that in selected older patients, an intensification of the reduce intensity conditioning regimens may be effective at treating patients with active disease at time of transplantation. ${ }^{25,26}$ Unfortunately, the heterogeneity of the conditioning regimens within the EBMT registry database limits any more detailed analysis of this relationship.

While only $31 \%$ of patients in the cohort had available cytogenetic data, the presence of poor-risk cytogenetic features was associated with significantly inferior outcomes on multivariate analysis. There were however notable differences in the patient demographics of this group when compared with patients without any available cytogenetic information, and as such the cytogenetic findings of this study may not be extrapolated for the whole cohort of patients. Nevertheless, this observation highlights the prognostic significance of pretransplant cytogenetic evaluation and the need to consider specific treatment strategies in patients with poor-risk cytogenetics.

Specific questions need to be addressed with regards to the role of both induction chemotherapy, and dose-intensified reduced intensity conditioning, and their associated toxicity in older patients under consideration for allogeneic transplantation. Our study indicates that in older patients with advanced disease stage at transplantation, alternative treatment options or novel treatment regimens should be considered. The recent discovery of novel immunomodulatory and disease modifying agents has expanded the treatment options for a 
subset of patients with MDS. ${ }^{27-29}$ While the use of these agents, do not result in a long-term cure, several recent studies have indicated the feasibility of these agents in being incorporated into either the pretransplant induction regimen, or as part of the post-transplantation maintenance protocol. ${ }^{30}$ Given the relatively high relapse rates in these patients, the role of low toxicity agents as part of a pre-emptive maintenance program warrants further investigation.

The other major variable influencing outcomes in our study is the choice of donor; allografting with an HLA-matched sibling donor being associated with significantly reduced NRM when compared with the use of an unrelated donor. Early registry analyses on the results of MDS patients transplanted with unrelated donors (median recipient age $<38$ years) demonstrated a 2 -year NRM of $54 \%$ to $57 \%$. ${ }^{2,3}$ Advances in supportive care and conditioning regimens have contributed to the comparatively improved NRM in this cohort $(36 \%$ at 4 years). Particularly in the context of RIC HSCT, various centers have recently reported on the use of both sibling and unrelated donors with comparable outcomes. ${ }^{21,31}$ However, our data suggest that in older patients with MDS, the choice of stem-cell donor remains a point of consideration, although both types of donors result in significant long-term survival even in patients older than 60 years.

In summary, this study demonstrates that allogeneic HSCT is a potential curative therapeutic option for many older patients with MDS and that disease stage at time of transplantation, but not age, remains the most important factor influencing subsequent outcomes. In addition, the choice of stem-cell donor remains a significant consideration for allogeneic transplantation within this age group of pa- tients. Given the increasing choice of options within the field of MDS transplantation, an individualized approach should be adopted in determining specific treatment strategies toward the treatment of a patient with MDS.

\section{AUTHORS' DISCLOSURES OF POTENTIAL CONFLICTS OF INTEREST}

The author(s) indicated no potential conflicts of interest.

\section{AUTHOR CONTRIBUTIONS}

Conception and design: ZiYi Lim, Nicolaus Kroger, Ghulam J. Mufti, Theo M. de Witte

Provision of study materials or patients: ZiYi Lim, Rodrigo Martino, Jürgen Finke, Andrea Bacigalupo, Dietrich Beelen, Agnes Devergie, Emilio Alessandrino, Roel Willemze, Tapani Ruutu, Marc Boogaerts, Michele Falda, Jean-Pierre Jouet, Dietger Niederwieser, Nicolaus Kroger, Ghulam J. Mufti, Theo M. de Witte

Collection and assembly of data: ZiYi Lim, Anja van Biezen,

Theo M. de Witte

Data analysis and interpretation: ZiYi Lim, Ronald Brand, Anja van Biezen, Theo M. de Witte

Manuscript writing: ZiYi Lim, Dietrich Beelen, Ghulam J. Mufti, Theo M. de Witte

Final approval of manuscript: ZiYi Lim, Ronald Brand, Rodrigo Martino, Jürgen Finke, Dietrich Beelen, Roel Willemze, Dietger Niederwieser, Nicolaus Kroger, Ghulam J. Mufti, Theo M. de Witte

\section{REFERENCES}

1. Anderson JE, Anasetti C, Appelbaum FR, et al: Unrelated donor marrow transplantation for myelodysplasia (MDS) and MDS-related acute myeloid leukaemia. Br J Haematol 93:59-67, 1996

2. Arnold $R$, de Witte $T$, van Biezen $A$, et al: Unrelated bone marrow transplantation in patients with myelodysplastic syndromes and secondary acute myeloid leukemia: An EBMT survey. European Blood and Marrow Transplantation Group Bone Marrow Transplant 21:1213-1216, 1998

3. Castro-Malaspina $H$, Harris RE, Gajewski J, et al: Unrelated donor marrow transplantation for myelodysplastic syndromes: Outcome analysis in 510 transplants facilitated by the National Marrow Donor Program. Blood 99:1943-1951, 2002

4. Copelan EA, Penza SL, Elder PJ, et al: Analysis of prognostic factors for allogeneic marrow transplantation following busulfan and cyclophosphamide in myelodysplastic syndrome and after leukemic transformation. Bone Marrow Transplant 25: 1219-1222, 2000

5. Cutler CS, Lee SJ, Greenberg P, et al: A decision analysis of allogeneic bone marrow transplantation for the myelodysplastic syndromes: Delayed transplantation for low-risk myelodysplasia is associated with improved outcome. Blood 104:579585,2004

6. Martino R, lacobelli $S$, Brand R, et al: Retrospective comparison of reduced-intensity conditioning and conventional high-dose conditioning for allogeneic hematopoietic stem cell transplantation using HLA-identical sibling donors in myelodysplastic syndromes. Blood 108:836-846, 2006

7. Yakoub-Agha I, de La Salmoniere P, Ribaud P et al: Allogeneic bone marrow transplantation for therapy-related myelodysplastic syndrome and acute myeloid leukemia: A long-term study of 70 patientsreport of the French society of bone marrow transplantation. J Clin Oncol 18:963-971, 2000

8. Slavin S, Nagler A, Naparstek E, et al: Nonmyeloablative stem cell transplantation and cell therapy as an alternative to conventional bone marrow transplantation with lethal cytoreduction for the treatment of malignant and nonmalignant hematologic diseases. Blood 91:756-763, 1998

9. Deeg HJ, Storer B, Slattery JT, et al: Conditioning with targeted busulfan and cyclophosphamide for hemopoietic stem cell transplantation from related and unrelated donors in patients with myelodysplastic syndrome. Blood 100:1201-1207, 2002

10. Gratwohl A, Baldomero H, Passweg J, et al: Hematopoietic stem cell transplantation for hematological malignancies in Europe. Leukemia 17:941959, 2003

11. Alyea EP, Kim HT, Ho V, et al: Impact of conditioning regimen intensity on outcome of allogeneic hematopoietic cell transplantation for advanced acute myelogenous leukemia and myelodysplastic syndrome. Biol Blood Marrow Transplant 12:10471055, 2006

12. de Lima M, Anagnostopoulos A, Munsell M, et al: Nonablative versus reduced-intensity conditioning regimens in the treatment of acute myeloid leukemia and high-risk myelodysplastic syndrome: Dose is relevant for long-term disease control after allogeneic hematopoietic stem cell transplantation. Blood 104:865-872, 2004

13. Crawley $C$, lacobelli $S$, Bjorkstrand B, et al: Reduced-intensity conditioning for myeloma: Lower nonrelapse mortality but higher relapse rates compared with myeloablative conditioning. Blood 109 3588-3594, 2007
14. Martino $R$, Caballero MD, Simon JA, et al: Evidence for a graft-versus-leukemia effect after allogeneic peripheral blood stem cell transplantation with reduced-intensity conditioning in acute myelogenous leukemia and myelodysplastic syndromes. Blood 100:2243-2245, 2002

15. de Witte T, Hermans J, Vossen J, et al: Haematopoietic stem cell transplantation for patients with myelo-dysplastic syndromes and secondary acute myeloid leukaemias: A report on behalf of the Chronic Leukaemia Working Party of the European Group for Blood and Marrow Transplantation (EBMT). Br J Haematol 110:620-630, 2000

16. Alyea EP, Kim HT, Ho V, et al: Comparative outcome of nonmyeloablative and myeloablative allogeneic hematopoietic cell transplantation for patients older than 50 years of age. Blood 105:1810-1814, 2005

17. Alamo J, Shahjahan M, Lazarus HM, et al: Comorbidity indices in hematopoietic stem cell transplantation: A new report card. Bone Marrow Transplant 36:475-479, 2005

18. Artz AS, Pollyea DA, Kocherginsky $M$, et al: Performance status and comorbidity predict transplant-related mortality after allogeneic hematopoietic cell transplantation. Biol Blood Marrow Transplant 12:954-964, 2006

19. Sorror ML, Maris MB, Storb R, et al: Hematopoietic cell transplantation (HCT)-specific comorbidity index: A new tool for risk assessment before allogeneic HCT. Blood 106:2912-2919, 2005

20. Lim ZY, Ho AY, Ingram W, et al: Outcomes of alemtuzumab-based reduced intensity conditioning stem cell transplantation using unrelated donors for myelodysplastic syndromes. $\mathrm{Br} \mathrm{J}$ Haematol 135: 201-209, 2006 
21. Scott BL, Sandmaier BM, Storer B, et al: Myeloablative vs nonmyeloablative allogeneic transplantation for patients with myelodysplastic syndrome or acute myelogenous leukemia with multilineage dysplasia: A retrospective analysis. Leukemia 20:128-135, 2006

22. Scott BL, Storer B, Loken MR, et al: Pretransplantation induction chemotherapy and posttransplantation relapse in patients with advanced myelodysplastic syndrome. Biol Blood Marrow Transplant 11:65-73, 2005

23. De Witte T, Muus $P$, De Pauw B, et al: Intensive antileukemic treatment of patients younger than 65 years with myelodysplastic syndromes and secondary acute myelogenous leukemia. Cancer 66:831-837, 1990

24. Parker JE, Pagliuca A, Mijovic A, et al: Fludarabine, cytarabine, G-CSF and idarubicin (FLAG-IDA) for the treatment of poor-risk myelodysplastic syndromes and acute myeloid leukaemia $\mathrm{Br} \mathrm{J}$ Haematol 99:939-944, 1997

25. Bertz H, Potthoff K, Finke J: Allogeneic stemcell transplantation from related and unrelated donors in older patients with myeloid leukemia. J Clin Oncol 21:1480-1484, 2003

26. Spyridonidis A, Bertz H, Ihorst G, et al: Hematopoietic cell transplantation from unrelated donors as an effective therapy for older patients ( $>$ or $=60$ years) with active myeloid malignancies. Blood 105 : 4147-4148, 2005

27. List A, Dewald G, Bennett J, et al: Lenalidomide in the myelodysplastic syndrome with chromosome 5q deletion. N Engl J Med 355:1456-1465, 2006

28. Silverman LR, Mufti GJ: Methylation inhibitor therapy in the treatment of myelodysplastic syndrome. Nat Clin Pract Oncol 2:S12-S23, 2005 (suppl 1)

29. Kantariian $H$, Issa JP, Rosenfeld CS, et al: Decitabine improves patient outcomes in myelodysplastic syndromes: Results of a phase III randomized study. Cancer 106:1794-1803, 2006

30. Jabbour E, Giralt S, Kantarjian H, et al: Lowdose azacitidine after allogeneic stem cell transplantation for acute leukemia. Cancer 115:1899-1905, 2009

31. Ho AY, Pagliuca A, Kenyon M, et al: Reducedintensity allogeneic haematopoietic stem cell transplantation for myelodysplastic syndrome and acute myeloid leukaemia with multilineage dysplasia using fludarabine, busulphan and alemtuzumab (CAMPATH-1H)(FBC) conditioning. Blood 104:16161623,2004

Journal of Clinical Oncology - The ideal place to publish your research

- Impact Factor of 17.157: JCO's published articles were cited 97,639 times and accounted for fully $9.7 \%$ of all oncology journal citations in 2008.

- Maximum Exposure: More than 25,000 of the world's leading oncology professionals receive JCO and more than 180,000 unique visitors per month visit jco.org.

- Outstanding Reputation: With an acceptance rate of just $20 \%, J C O$ publishes only the very best articles in the field.

- International Coverage: JCO is available globally in 28 countries and in 15 international editions.

To submit a manuscript, visit submit.jco.org.
- Rapid Turnaround: JCO averages just 9 weeks from final manuscript acceptance to online publication.

- No Exclusivity Clause: JCO authors may reproduce or reuse their own material published in $J C O$ for educational purposes at no charge.

- No Submission Charges: JCO has no submission, color, or page charges. 